\title{
Rare Earth Ion Implantation in GaN: Damage Formation and Recovery
}

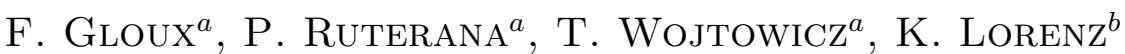 \\ AND E. Alves ${ }^{b}$ \\ ${ }^{a}$ SIFCOM, UMR 6176, CNRS-ENSICAEN, 14050 Caen, France \\ ${ }^{b}$ Instituto Tecnológico e Nuclear, EN10, 2686-953 Sacavém, Portugal
}

Rare earth ions implanted GaN has been investigated by transmission electron microscopy versus the fluence, using Er, Eu or Tm ions at $150 \mathrm{keV}$ or $300 \mathrm{keV}$ and at room temperature. Point defect clusters and stacking faults are generated from low fluences $\left(7 \times 10^{13} \mathrm{at} / \mathrm{cm}^{2}\right)$, their density increases with the fluence up to the formation of a highly disordered layer at the surface. This highly disordered layer is observed from a threshold fluence of $3 \times 10^{14}$ at $/ \mathrm{cm}^{2}$ at $150 \mathrm{keV}$ and $3 \times 10^{15}$ at $/ \mathrm{cm}^{2}$ at $300 \mathrm{keV}$, and appears to be composed of voids and misoriented nanocrystallites. Its thickness rapidly increases with the fluence, and then saturates. Both basal and prismatic stacking faults were observed. Basal stacking faults are $I_{1}$ in majority, but $E$ or $I_{2}$ have also been identified. $I_{1}$ basal stacking faults propagate easily through GaN by folding from basal to prismatic planes. Channelling implantation, increasing the implantation temperature from room temperature to $500^{\circ} \mathrm{C}$, or implanting through a $10 \mathrm{~nm}$ thick AlN cap reduce the crystallographic damage, particularly by retarding the formation of the highly disordered layer. Implanting through the AlN cap allows the highly disordered layer formation threshold fluence to be increased by one order of magnitude, as well as the annealing at high temperature $\left(1300^{\circ} \mathrm{C}\right)$ which brings about a strong optical activation of the rare earths.

PACS numbers: 61.72.Vv, 61.72.Nn, 68.37.Lp, 81.05.-t

\section{Introduction}

For the last few years, rare earths (REs) doped GaN have received a large interest due to expected promising applications in optoelectronics and photonics. In situ GaN doping by molecular beam epitaxy has brought about important results: integration of $\mathrm{Eu}, \mathrm{Er}$, and Tm for full-color displays, laser action from Eu-doped GaN at room temperature (RT) $[1,2]$. Ion implantation is a competitive technique with many advantages, particularly the possibility to control the doped 
area, an easier lateral integration, or the formation of highly resistive regions which are needed for fabrication of GaN based electronic devices such as high electron mobility transistors $[3,4]$.

Therefore, determination of the optimized conditions for implantation and annealing of GaN is necessary in order to minimize the induced damage. For the as-implanted GaN, early studies [5-7] have reported on some typical features: planar defects, and an amorphous layer which formed on top of GaN. The planar defects have been observed to be the most characteristic defects in GaN bombarded with ions in a wide range of implantation conditions, independently of the ion, fluence, energy, and implantation temperature. The amorphous layer was reported to form for ion fluences exceeding some critical value, at RT or liquid nitrogen temperature $[6,7]$. However, recent results have reported not only on amorphous material in this layer but also on some crystalline parts [8-10]. There have also been a few investigations on nature of the induced planar defects and their formation mechanism [11]. The role, they may play in the formation of the amorphous or at least highly disordered layer (HDL) at the surface, needs to be determined since planar defects were suggested to constitute the "nucleation site" for the "amorphization" of the surface [6].

As has been pointed out, implanted GaN is difficult to anneal [7]; by $1100^{\circ} \mathrm{C}$, the GaN surface already suffers from strong dissociation [12]. One way of getting around this problem has been to try to reduce the damage at the implantation stage. Channelling of ions [13, 14], implantation at temperature higher than RT [15] and/or through a thin AlN cap grown on GaN top [10] were shown to decrease the damage amount, particularly by retarding the formation of the HDL. Especially, implanting and annealing with a thin AlN cap has been recently demonstrated to permit annealing temperatures as high as $1300^{\circ} \mathrm{C}$, with a 30 times increase of the luminescence emission from $1000^{\circ} \mathrm{C}$ to $1300^{\circ} \mathrm{C}$ [16].

Structural investigations are needed to understand the influence on the density, nature, and distribution of defects, of the different solutions that can be used to decrease the damage. In this work, structural studies of the induced damage in RE implanted GaN have been carried out by transmission electron microscopy (TEM) and Rutherford backscattering spectroscopy/channeling (RBS/C). The influence of implantation parameters: fluence, implantation direction, implantation temperature, the use of a $10 \mathrm{~nm}$ epitaxial AlN cap, has been analyzed in as-implanted GaN. For annealed AlN capped GaN, the impact of the annealing temperature has been investigated.

\section{Experimental}

$2 \mu \mathrm{m}$ thick GaN layers grown on (0001) sapphire by metal organic vapour phase epitaxy (MOVPE) were implanted using Er, Eu or Tm RE ions, either at $150 \mathrm{keV}$ or $300 \mathrm{keV}$, at RT or $500^{\circ} \mathrm{C}$, with fluences from $5 \times 10^{13} \mathrm{at} / \mathrm{cm}^{2}$ to $2 \times 10^{16} \mathrm{at} / \mathrm{cm}^{2}$. On top of some GaN layers, a $10 \mathrm{~nm}$ AlN ultrathin layer had 
been grown at the end of the MOVPE. These samples were implanted at $300 \mathrm{keV}$, RT, with fluences between $2 \times 10^{15} \mathrm{at} / \mathrm{cm}^{2}$ and $4 \times 10^{16} \mathrm{at} / \mathrm{cm}^{2}$. Annealings were performed on AlN capped GaN with temperatures ranging between 1000 and $1300^{\circ} \mathrm{C}$. They were carried out in a conventional furnace under a nitrogen overpressure of $4 \times 10^{5} \mathrm{~Pa}$ with holding times of $20 \mathrm{~min}$.

Conventional TEM (CTEM) and high resolution TEM (HRTEM) were performed on cross-sections thinned down to $100 \mu \mathrm{m}$ by mechanical grinding and dimpled down to $15 \mu \mathrm{m}$. The electron transparency was achieved by ion milling at $5 \mathrm{kV}$ using the GATAN precision ion polisher (PIPS) at an incidence angle of $5^{\circ}$. CTEM was carried out with a JEOL 2010 microscope operating at $200 \mathrm{keV}$ and HRTEM was performed in a Topcon 002B instrument operating at $200 \mathrm{keV}$ with a point resolution of $0.18 \mathrm{~nm}$. SEM imaging was carried out with a JEOL JSM-6400 microscope.

RBS/C studies were performed with a $1 \mathrm{~mm}$ diameter collimated beam of $2 \mathrm{MeV} \mathrm{He}{ }^{+}$ions. The backscattered particles were detected at $140^{\circ}$ and close to $180^{\circ}$ with respect to the incoming beam direction using silicon surface barrier detectors with resolutions of 13 and $16 \mathrm{keV}$, respectively, located in the standard IBM geometry.

\section{Results}

\subsection{Evolution of the implantation damage versus the fluence}

The nature and distribution of the implantation damage have been investigated for fluences from $7 \times 10^{13}$ at $/ \mathrm{cm}^{2}$ to $2 \times 10^{16}$ at $/ \mathrm{cm}^{2}$. CTEM observations performed on cross-sectional samples with dark-field weak-beam conditions, using $g$ vectors equal to 0002 and $10 \overline{1} 0$, give information on atomic displacements along the $c$-axis and in the basal plane, respectively. In the 0002 images which reveal point defect clusters, there is an increase in density with fluence as can be seen in Fig. 1a and b: point defect clusters, corresponding to the bright spots, become larger and are visible deeper in the GaN layer. This increase in the damage density with the fluence was seen to occur up to $4 \times 10^{15}$ at $/ \mathrm{cm}^{2}$, corresponding to the formation onset at the GaN surface of the HDL that was reported by [8-10]. For fluences above the threshold for the HDL formation, the point defect clusters density saturates. The HDL observed in bright-field CTEM for the $5 \times 10^{15} \mathrm{Tm} / \mathrm{cm}^{2}$ fluence is exhibited in Fig. 2a. Figure $2 \mathrm{~b}$ shows the structure of this HDL imaged in HRTEM along the [1120] GaN zone axis. It appears to be a mixture of nanocrystalline patches (black arrows), rotated from each other and highly misoriented with respect to the less damaged still monocrystalline GaN, and of voids (white arrows). The mean diameter of the nanocrystallites is about $3 \mathrm{~nm}$. No amorphous material is clearly evidenced by this image. Previous results have reported on highly damaged layers made of "broken crystals and amorphous in nanometer sizes" [8], "amorphous zones and small crystalline domains that are arranged in a random orientation" [9], whereas a "nanocrystalline layer" composed 


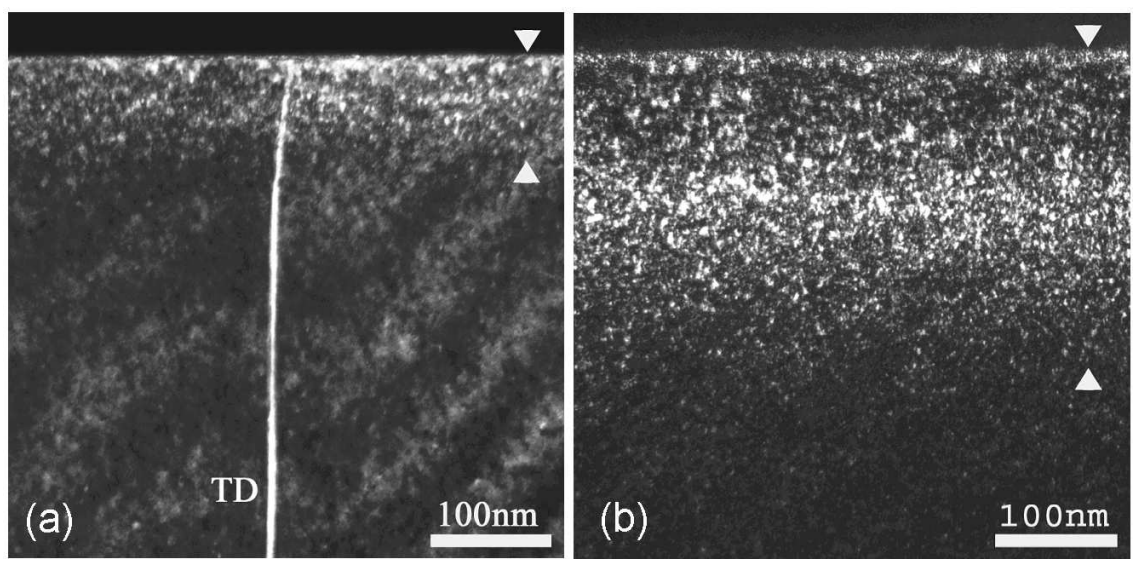

Fig. 1. The point defect cluster distribution in GaN implanted at $300 \mathrm{keV}$ and RT, imaged with dark-field [0002] weak-beam conditions for: (a) fluence of $7 \times 10^{13} \mathrm{Eu} / \mathrm{cm}^{2}$ (a threading dislocation (TD) is visible on the micrograph); (b) fluence of $2 \times 10^{15} \mathrm{Er} / \mathrm{cm}^{2}$.

of "voids and nanocrystals" has been observed by Wojtowicz et al. [10]. The bright contrasts in our HRTEM images (white arrows in Fig. 2b) may be attributed to voids superimposed with nanocrystallites that are misoriented with respect to the $[11 \overline{2} 0]$ zone axis.

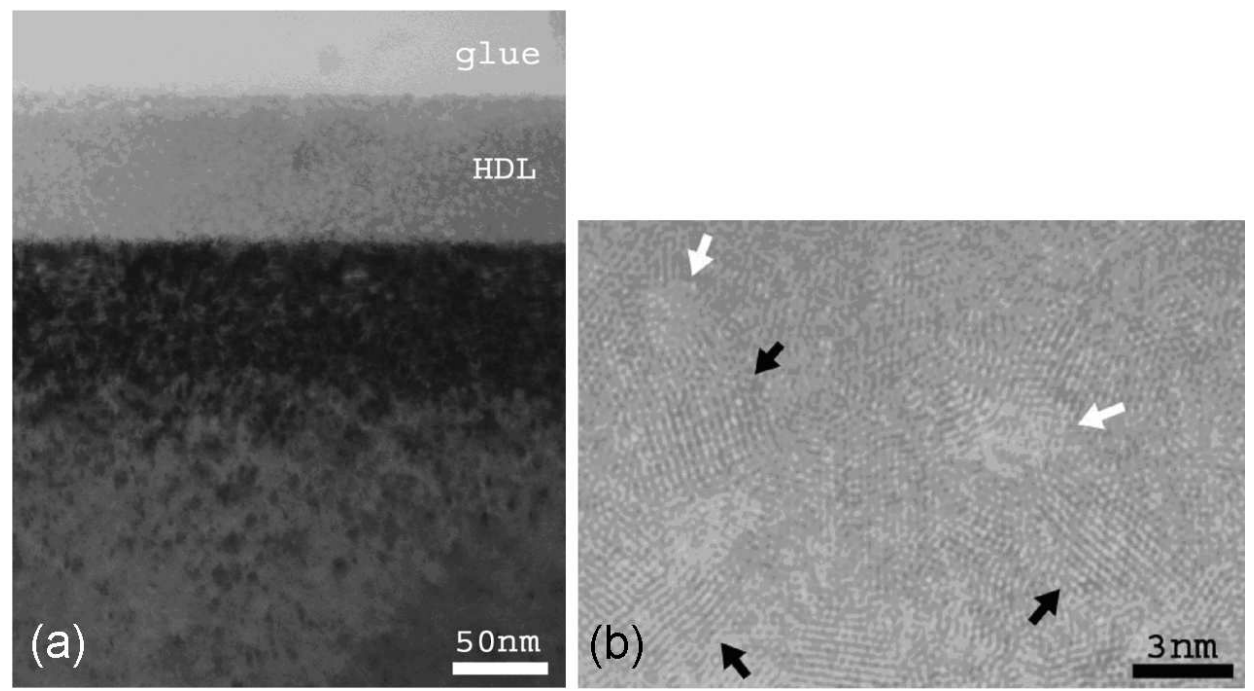

Fig. 2. (a) The high disordered layer at the GaN surface, for implantation at $300 \mathrm{keV}$ and RT with $5 \times 10^{15} \mathrm{Tm} / \mathrm{cm}^{2}$; (b) HRTEM image of the HDL along the [11 $\left.\overline{2} 0\right]$ zone axis; its structure is a mixture of voids (white arrows) and misoriented nanocrystals (black arrows). 


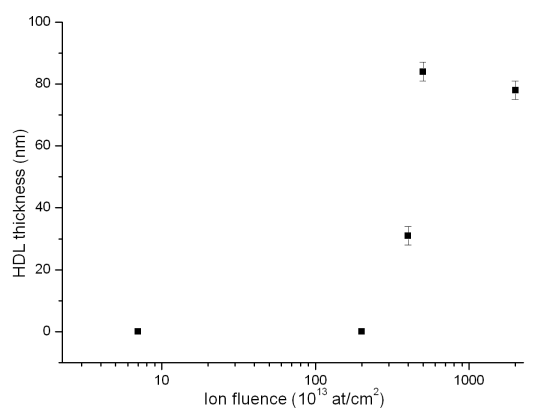

Fig. 3. HDL thickness versus implanted rare earth ion fluence into GaN at $300 \mathrm{keV}$ and RT.

As deduced from CTEM, the HDL thickness increases sharply from zero between $2 \times 10^{15}$ at $/ \mathrm{cm}^{2}$ and $4 \times 10^{15}$ at $/ \mathrm{cm}^{2}$ (Fig. 3), which is in agreement with corresponding RBS/C results [14]: the fluence at which the random level is reached is about $2.5 \times 10^{15} \mathrm{at} / \mathrm{cm}^{2}$. This indicates that a high level of disorder has accumulated at the GaN surface, and from this fluence, the HDL started to form. The evolution of the HDL thickness versus the fluence (Fig. 3) exhibits a rapid increase from the threshold value. Above $4 \times 10^{15} \mathrm{at} / \mathrm{cm}^{2}$, its thickness appears to saturate. Results for implantation at $150 \mathrm{keV}$ and RT exhibit the same behaviour: the formation of the HDL from the surface towards the volume with a fast increase in the thickness with the fluence and then saturation [14]. This process seems to be characteristic of damage build-up in GaN.

Planar defects are observed from the lowest fluences: at $7 \times 10^{13}$ at $/ \mathrm{cm}^{2}$, a few weak horizontal contrasts (arrows) are visible (Fig. 4a). (Let us note that in this figure, a series of horizontal fringes are visible in the first $10 \mathrm{~nm}$ at the
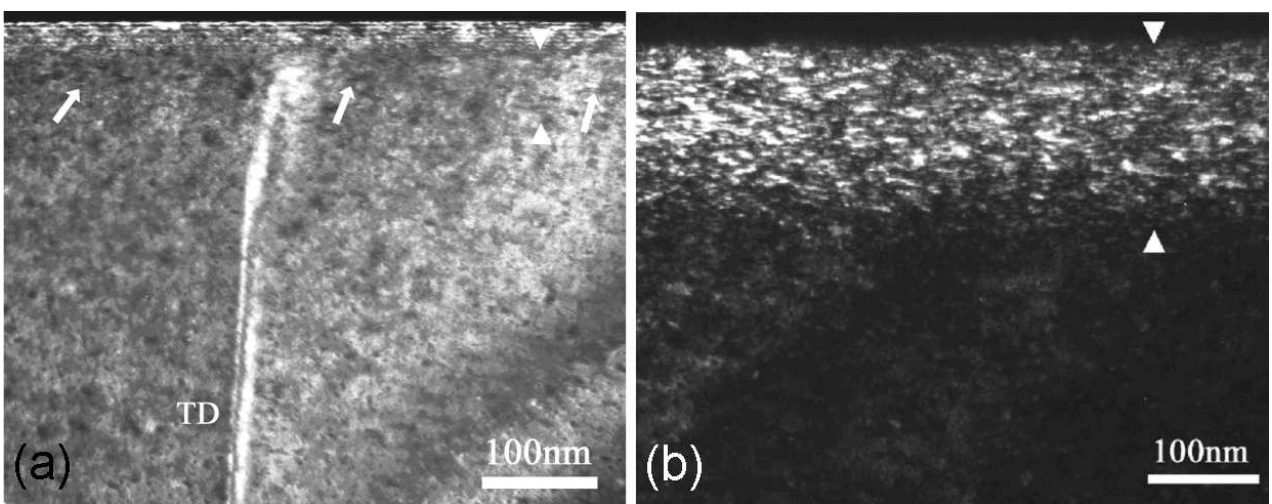

Fig. 4. Planar defects distribution in the damaged region (between short white arrows) of GaN implanted at $300 \mathrm{keV}$ and RT; dark-field weak-beam images taken with $g=10 \overline{10}$, for fluences of: (a) $7 \times 10^{13} \mathrm{Eu} / \mathrm{cm}^{2}$ (some basal stacking faults are indicated by long white arrows); (b) $2 \times 10^{15} \mathrm{Er} / \mathrm{cm}^{2}$. 
layer surface; these are thickness fringes due to the inclined sample surface). The density first increases with the fluence, as exhibited in the dark-field weak-beam images with $g=10 \overline{1} 0$ for the fluences $7 \times 10^{13}$ at $/ \mathrm{cm}^{2}$ and $2 \times 10^{15}$ at $/ \mathrm{cm}^{2}$ in Fig. $4 \mathrm{a}$ and b. A closer examination of the defects using HRTEM shows that not only basal stacking faults (BSFs) but also prismatic stacking faults (PSFs) have been generated, independently of the fluence. $I_{1}$ and $E$ BSFs have been identified (Fig. 5), as well as $I_{2}$ BSFs. The Table exhibits the density of BSFs by type, their relative percentage versus the fluence and the total BSFs density, deduced from HRTEM images. The total BSFs density increases with the fluence up to the HDL formation threshold, as roughly observed from the CTEM study; it then saturates. BSFs are $I_{1}$ in the majority, this stacking fault type has the lowest formation energy [17]. The formation of $I_{1}$ and $E$ BSFs implies the insertion of an extra half atomic plane, meaning that the BSFs may be related to interstitials. Increasing the fluence towards the HDL threshold, the $E$ fraction slightly increases.

TABLE

Type of BSFs and their density versus the implantation fluence.

\begin{tabular}{c|c|c|c|c|c|c|c|c|c|c|c}
\hline \hline $\begin{array}{c}\text { Fluence } \\
\left(\mathrm{cm}^{-2}\right)\end{array}$ & \multicolumn{3}{|c|}{$\begin{array}{c}\text { Relative } \\
\text { percentage }(\%)\end{array}$} & \multicolumn{3}{c|}{$\begin{array}{c}\text { BSF density }\left(10^{11} / \mathrm{cm}^{2}\right) \\
( \pm 3 \%)\end{array}$} & \multicolumn{3}{c}{$\begin{array}{c}\text { Distance between BSF (nm) } \\
( \pm 0.3 \mathrm{~nm})\end{array}$} \\
\cline { 2 - 11 } & $I_{1}$ & $I_{2}$ & $E$ & all types & $I_{1}$ & $I_{2}$ & $E$ & $I_{1}$ & $I_{2}$ & $E$ & all types \\
\hline $7 \times 10^{13}$ & 90 & 7 & 3 & 9.0 & 8.1 & 0.6 & 0.2 & 6.0 & 14.0 & 24.0 & 6.0 \\
$2 \times 10^{15}$ & 92 & 2 & 6 & 34 & 31 & 1.0 & 2.0 & 4.0 & 14.0 & 12.5 & 4.0 \\
$5 \times 10^{15}$ & 90 & 4 & 6 & 33 & 30 & 1.0 & 2.0 & 4.0 & 9.0 & 6.5 & 4.0 \\
$2 \times 10^{16}$ & 91 & 4 & 5 & 38 & 34 & 1.5 & 2.0 & 3.5 & 17.5 & 9.0 & 3.5
\end{tabular}

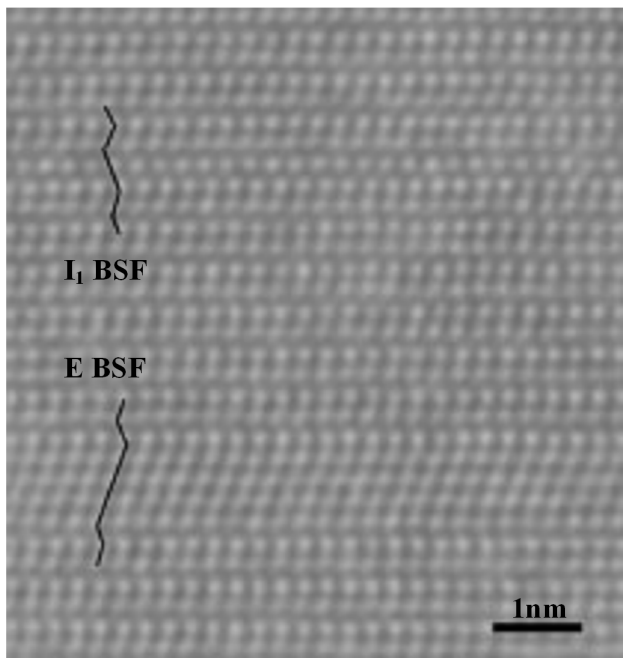

Fig. 5. Basal stacking faults (BSFs) induced in GaN by implantation at $300 \mathrm{keV}$ and RT, for the $2 \times 10^{15} \mathrm{Er} / \mathrm{cm}^{2}$ fluence: $I_{1}$ and $E$ BSFs are shown. 
PSFs are observed for all the fluences. They are found to be the way for the $I_{1}$ BSFs to easily change their level and propagate through the implanted layer: the $I_{1}$ BSF folds to a $\{11 \overline{2} 0\}$ prismatic plane through a PSF then goes into another basal plane. Different configurations of climbing process have been seen to take place: the basal plane displacement vectors for the $I_{1}$ BSFs related by the PSF can be either equal or opposite. Figure $6 \mathrm{a}$ exhibits the first case. The contrast in the prismatic plane corresponds to the Drum atomic configuration of the PSF (between the black arrows) with displacement vector $\boldsymbol{D}=\frac{1}{2}[10 \overline{1} 1]$ [18]. The same

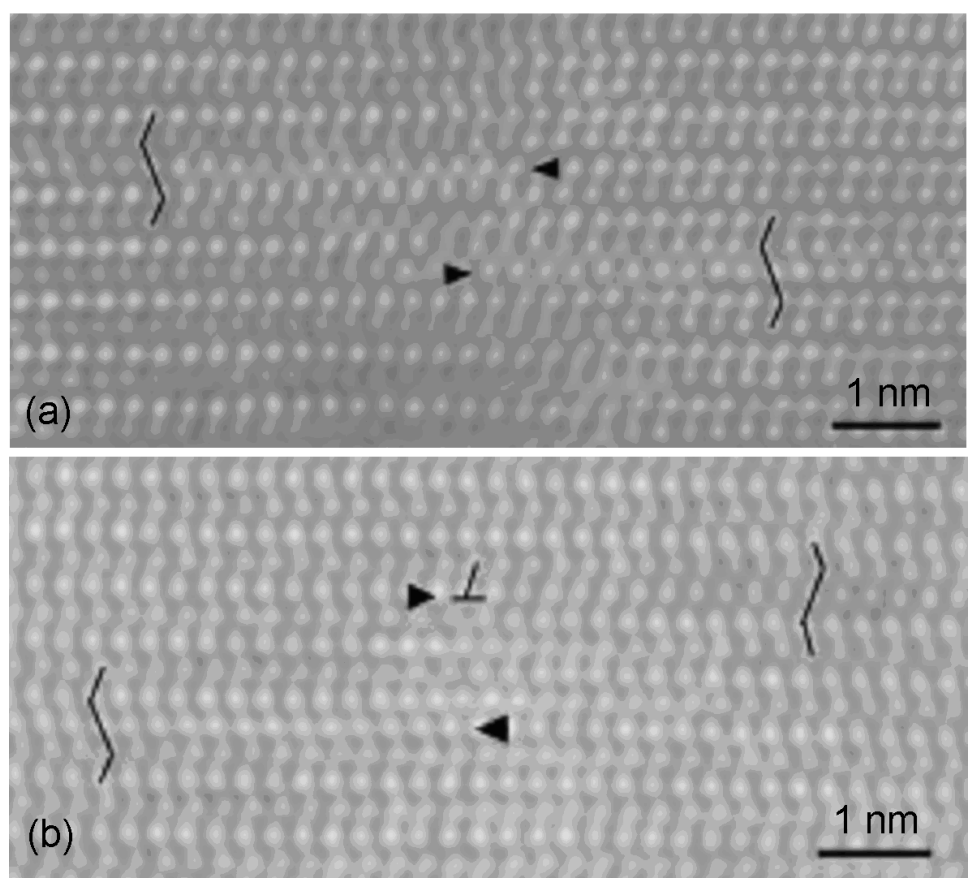

Fig. 6. $\quad I_{1}$ BSFs folding into the prismatic plane with the Drum atomic configuration; crossing by the PSF is indicated by black arrows. (a) The $I_{1}$ BSF goes into the prismatic plane and back into the basal plane keeping the same displacement vector. (b) The displacement in the basal plane is opposite on the two sides of the PSF.

$I_{1}$ BSF has undergone a climb process by folding in the prismatic plane and back, this process implies a double change of displacement vectors: $\frac{1}{6}[10 \overline{1} 1]$ then $\frac{1}{2}[10 \overline{1} 1]$ and back. In Fig. 6b, the two displacement vectors are opposite but the PSF exhibits again the Drum configuration, with the presence of an additional (1010) half plane this time. The two related $I_{1}$ BSFs have probably interacted through the PSF formation. In the investigated cases, PSF extensions are small, less than 3c. In fact $I_{1}$ basal SFs have been observed to almost systematically fold from the basal to the prismatic plane by PSFs. 


\subsection{Reducing the implantation induced damage}

In order to reduce the amount of these defects and particularly the formation of the high disordered layer, a number of solutions have recently been proposed $[13,15]$.

\subsubsection{Channelled implantation}

In their work, Pipeleers et al. have recently pointed out a reduction of induced damage using channelled implantation of Er ions in GaN at $170 \mathrm{keV}$ and RT [13]: higher fluences could be implanted before reaching the same damage level as in the random geometry. Even if the influence seemed to be reduced for fluences above the HDL threshold (expected slightly higher than the $3 \times 10^{14} \mathrm{at} / \mathrm{cm}^{2}$ value at $150 \mathrm{keV}$ and RT [14]), channelled implantation was observed to lead to drastically decreased defect concentration up to ion fluence of $1 \times 10^{15} \mathrm{at} / \mathrm{cm}^{2}$. Thus, channelling appears to be an efficient and achievable way to minimize the implantation damage level, since the critical angle of this configuration is relatively large, about $6-7^{\circ}[13]$. However, as noticed by Lorenz et al. [14], although the crystallographic damage is reduced, the activation of the RE ions is still necessary and high temperature annealing is required.

\subsubsection{Implantation at high temperature: $500^{\circ} \mathrm{C}$}

Increasing the ion implantation temperature seems to be also an appropriate way to reduce the damage amount, considering that dynamic annealing and annihilation of interstitials are most efficient at higher temperature. Comparison of $\mathrm{RBS} / \mathrm{C}$ spectra obtained for the implantation is performed at RT and $500^{\circ} \mathrm{C}$ for a fluence of $5 \times 10^{15} \mathrm{Tm} / \mathrm{cm}^{2}$, i.e. above the HDL formation threshold at $150 \mathrm{keV}$ and RT, first showed that, if the implantation damage at $500^{\circ} \mathrm{C}$ is slightly higher in the volume of the implanted GaN, the damage level at the surface does not reach the random level [14].

Measuring RBS/C spectra versus fluence for implantation at $300 \mathrm{keV}$ and $500^{\circ} \mathrm{C}$ pointed out that the damage level remains well below the random one in a fluence range of $7 \times 10^{14}-7 \times 10^{15} \mathrm{Eu} / \mathrm{cm}^{2}$ [16]. It was shown that implanting at $500^{\circ} \mathrm{C}$ efficiently suppresses the $\mathrm{HDL}$ formation up to the fluence of $7 \times 10^{15} \mathrm{Eu} / \mathrm{cm}^{2}$ at least. Moreover, the damage distribution in the GaN volume and the Tm profile are broader at $500^{\circ} \mathrm{C}$, and $\mathrm{Tm}$ ions and damages extend further in the GaN layer. This behaviour has been related to an increase in defect diffusion during the high temperature implantation and to an enhanced channelling due to the HDL formation inhibition at the layer surface, in the case of $150 \mathrm{keV}$ Tm implantation [19].

However implantation at elevated temperature is reported to imply anomalous surface erosion [20], and the damage is not completely removed, so, a high temperature annealing step is also necessary. 


\subsubsection{Implantation through an ultrathin AlN cap}

A micrograph of a GaN layer implanted with $5 \times 10^{15} \mathrm{Tm} / \mathrm{cm}^{2}$ at $300 \mathrm{keV}$ and RT through a $10 \mathrm{~nm}$ AlN cap grown on top is shown in Fig. 7. For comparison, the structure of a GaN layer implanted with the same conditions without AlN cap can be seen in Fig. 2. In the case of fluence above the HDL threshold, as shown in these bright-field TEM images, it is clear that implanting through the AlN inhibits the damage build-up at the sample surface and prevents the HDL formation. Without the AlN cap, implantation leads to the formation of a $85 \mathrm{~nm}$ thick HDL (Fig. 2). When implantation is performed through the AlN cap, no HDL is observed at the GaN surface (Fig. 7).

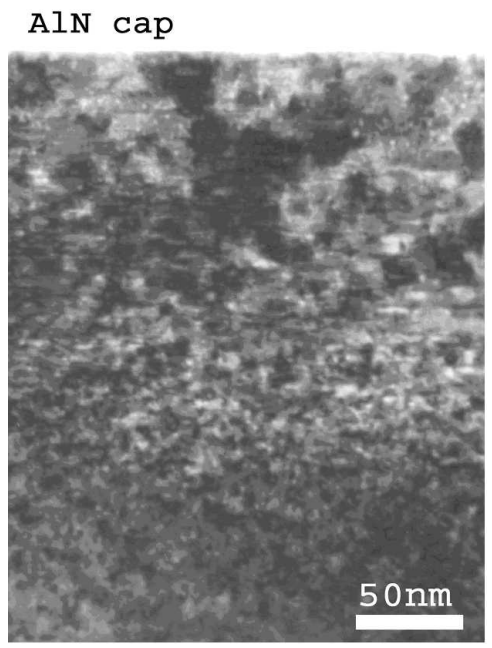

Fig. 7. Bright-field CTEM image of the surface of GaN capped with a $10 \mathrm{~nm}$ AlN layer surface, implanted at $300 \mathrm{keV}$ and RT, with $5 \times 10^{15} \mathrm{Tm} / \mathrm{cm}^{2}$; comparison with Fig. 2 points out the HDL inhibition provided by the AlN cap.

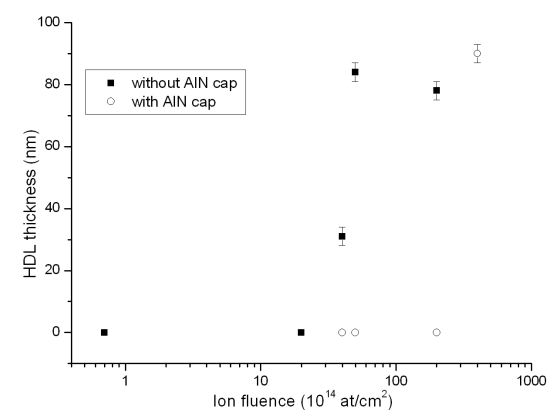

Fig. 8. HDL thickness versus implanted rare earth ion fluence at $300 \mathrm{keV}$ and RT, for AlN capped and uncapped GaN layers. 
We have investigated the HDL formation in AlN capped GaN samples versus the fluence, from $4 \times 10^{15} \mathrm{Eu} / \mathrm{cm}^{2}$ to $4 \times 10^{16} \mathrm{Eu} / \mathrm{cm}^{2}$, at $300 \mathrm{keV}$ and RT. The results of HDL thickness determination versus the fluence are in Fig. 8; the values for implantation without AlN cap are also indicated. With the $10 \mathrm{~nm}$ AlN cap, the HDL build-up appears to follow the same behaviour as implantation through a bare GaN surface: the HDL is formed from a threshold fluence, and its thickness also rapidly increases. However, the HDL formation threshold is above $2 \times 10^{16} \mathrm{at} / \mathrm{cm}^{2}$. Therefore, the AlN cap allows one to gain one order of magnitude on the HDL formation threshold, which is a significant improvement. A HRTEM micrograph of an AlN capped GaN layer implanted with $2 \times 10^{16} \mathrm{Eu} / \mathrm{cm}^{2}$ at $300 \mathrm{keV}$ and RT (Fig. 9) shows that even up to this high fluence, no BSFs are generated in AlN.

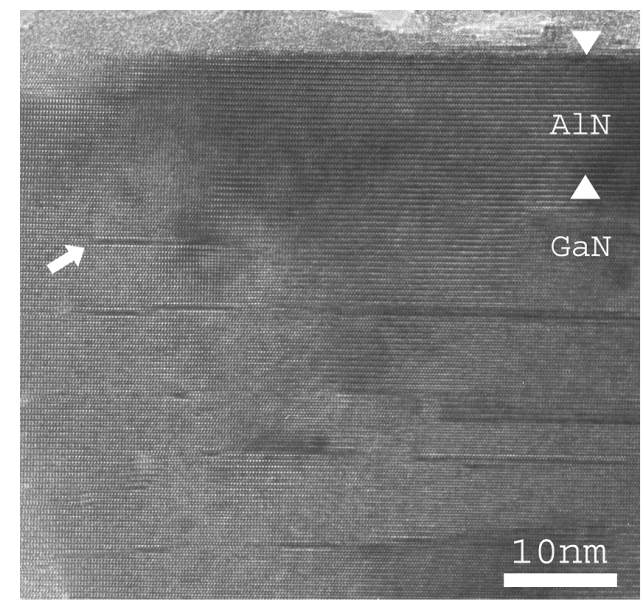

Fig. 9. A HRTEM micrograph of $10 \mathrm{~nm}$ AlN capped GaN implanted at $300 \mathrm{keV}$, RT, with a fluence of $2 \times 10^{16} \mathrm{Eu} / \mathrm{cm}^{2}$; the first visible BSF (white arrow) is inside the GaN layer, well below the AlN cap.

The first visible one (white arrow) is inside the GaN layer. In addition, the AlN surface remains flat, unlike the uncapped GaN surface, which becomes rough after implantation; this difference has been observed for all the fluences [21]. This high resistance provided by the $10 \mathrm{~nm}$ AlN cap may be related to the investigation by Kucheyev et al. of $2 \mu \mathrm{m}$ thick AlN layers, implanted with $\mathrm{Au}$ ions at $300 \mathrm{keV}$, $\mathrm{RT}$, with fluences ranging from $2 \times 10^{15}$ at $/ \mathrm{cm}^{2}$ to $4 \times 10^{16} \mathrm{at} / \mathrm{cm}^{2}$ [22]. Their observation was explained by an efficient dynamic annealing of defects during implantation into AlN.

\subsection{Protection against the annealing induced dissociation}

\subsubsection{Implantation and annealing through the AlN cap}

Recent results have demonstrated that temperatures as high as $1300^{\circ} \mathrm{C}$ can be reached for annealing AlN capped GaN layers, leading to an improvement of the 
optical activity of the RE implanted GaN system. Measuring cathodoluminescence (CL) spectra at RT for AlN capped layers implanted at $300 \mathrm{keV}$ and RT with $1 \times 10^{15} \mathrm{Eu} / \mathrm{cm}^{2}$, when the annealing temperature is increased from $1000^{\circ} \mathrm{C}$ to $1300^{\circ} \mathrm{C}$, the maximum intensity of the $621 \mathrm{~nm}$ line increases by a factor of 30; for the integrated CL intensity of this line, the increase reaches a factor of 40 [16].

This improvement may be related to the decrease in the defect density in the annealed layer observed when the temperature increases. A recent HRTEM investigation of AlN capped GaN layers implanted at $300 \mathrm{keV}$, RT, with a fluence of $1 \times 10^{15} \mathrm{Eu} / \mathrm{cm}^{2}$, and annealed at $1000^{\circ} \mathrm{C}$ and $1200^{\circ} \mathrm{C}$, pointed out a decrease in the BSF density from $1000^{\circ} \mathrm{C}$ to $1200^{\circ} \mathrm{C}[10]$.

\subsubsection{Limit of the AlN cap protection}

Considering the previous results, using GaN samples capped with a thin AlN layer can be a promising way in the development of ion implantation in RE doped GaN based devices fabrication. However, it was shown that the used caps did not protect the whole GaN surface. Figure 10a is a SEM image of a $10 \mathrm{~nm}$ AlN capped GaN layer implanted at $300 \mathrm{keV}$ and RT with a fluence of $4 \times 10^{15} \mathrm{Tm} / \mathrm{cm}^{2}$, then annealed at $1300^{\circ} \mathrm{C}$. In between flat areas, which are a perfect crystalline material,

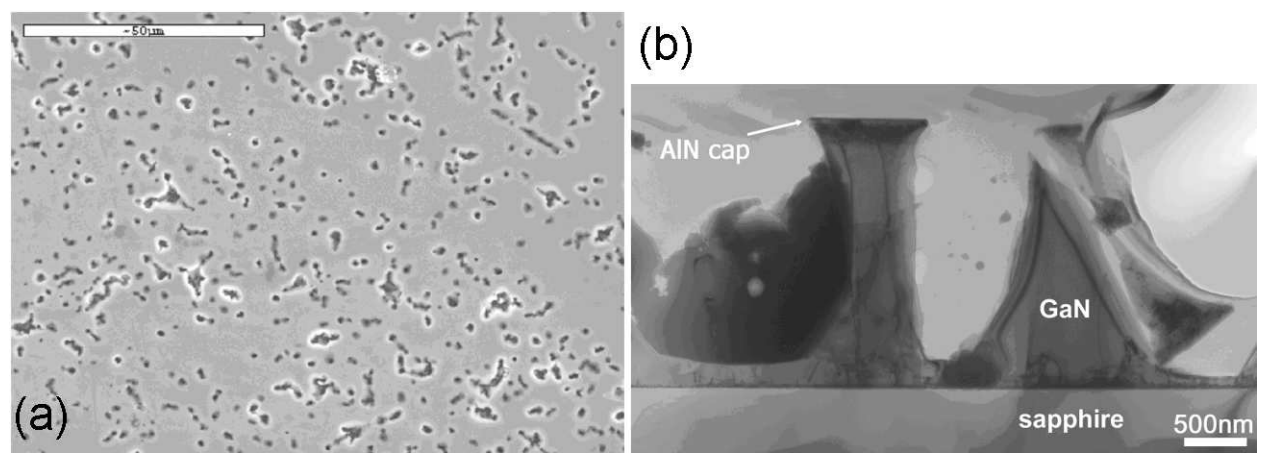

Fig. 10. (a) SEM micrograph of the surface of the $10 \mathrm{~nm}$ AlN capped GaN layer, implanted at $300 \mathrm{keV}$ and RT, with $4 \times 10^{15} \mathrm{Eu} / \mathrm{cm}^{2}$, after annealing at $1300^{\circ} \mathrm{C}$; (b) Cross-sectional TEM image of this sample, showing the strong dissociation of GaN.

a large amount of cracks can be seen at the sample surface. A cross-sectional TEM image of this sample is shown in Fig. 10b. It is clear that an explosive decomposition of the GaN layer has occurred; the cracks almost reach the GaN interface with the substrate. Our previous HRTEM investigation of the as-grown AlN cap structure has demonstrated that many pinholes are formed, as a way to relax the strain due to the $(-2.4 \%)$ lattice mismatch between AlN and GaN [21]. The thickness of the AlN cap, $10 \mathrm{~nm}$, is actually well above the critical thickness for relaxation, reported to be in the range 1-3 $\mathrm{nm}$ [23]. No misfit dislocations at the interface between AlN and GaN have been revealed by filtering the interface, so pinholes appear to provide a channel for strain relaxation. A recent study, using 
SEM and wavelength dispersive X-ray analysis, showed that GaN dissociation occurs via structural defects in the as-grown AlN layer [24]. During annealing, the AlN also decomposes, and, through the pinholes, this dissociation may reach the AlN/GaN interface, leading to the subsequent explosive decomposition of the underlying $\mathrm{GaN}$ at such high temperatures $\left(1300^{\circ} \mathrm{C}\right)$. In contrast, for the AlN areas without pinholes, the degradation did not reach the underlying $\mathrm{GaN}$ which gives rise to the flat areas shown in Fig. 10b. HRTEM images of the surface only reveal $5 \mathrm{~nm}$ surface roughness. Therefore, in comparison to the $1800 \mathrm{~nm}$ out of $2000 \mathrm{~nm}$ of GaN that are dissociated through the pinholes inside the AlN cap, AlN decomposes very slowly. In fact, the measured rate for the AlN decomposition is about 350 lower than for $\mathrm{GaN}$ at $1300^{\circ} \mathrm{C}$. The protective role of the AlN cap from the $\mathrm{GaN}$ dissociation during the $1300^{\circ} \mathrm{C}$ annealing is obvious, and explains the high optical activation of REs obtained after annealing at this temperature.

Considering this promising result, further work is carried out in order to achieve growth of pinhole-free AlN caps on GaN for electro-optical devices applications.

\section{Conclusion}

TEM investigation of GaN implanted with REs at $300 \mathrm{keV}$ and RT, versus fluence, showed that the damage build-up is governed by the formation of point defect clusters, and basal and prismatic stacking faults. Starting from a critical fluence, a HDL forms at the GaN surface and grows towards the volume. Among the ways to reduce the induced damage in GaN, implanting through a thin AlN cap appears to be attractive: the implantation can be performed with fluences ranging one order of magnitude higher before the HDL formation, i.e. up to about $2 \times 10^{16}$ at $/ \mathrm{cm}^{2}$. Moreover, using the AlN cap has been demonstrated to allow GaN to be annealed at temperature as high as $1300^{\circ} \mathrm{C}$, leading to a good optical activation of the REs. Provided pinhole-free AlN caps are grown on GaN, this is a promising procedure for the fabrication of devices in RE-doped GaN by ion implantation.

\section{References}

[1] D.S. Lee, A.J. Steckl, Appl. Phys. Lett. 80, 1888 (2002).

[2] J.H. Park, A.J. Steckl, Appl. Phys. Lett. 85, 4588 (2004).

[3] S.J. Pearton, J.C. Zolper, R.J. Shul, F. Ren, J. Appl. Phys. 86, 1 (1999).

[4] S.J. Pearton, F. Ren, A.P. Zhang, K.P. Lee, Mater. Sci. Engin. R 30, 55 (2000).

[5] C. Liu, B. Mensching, M. Zitler, K. Volz, B. Rauschenbach, Phys. Rev. B 57, 2530 (1998).

[6] S.O. Kucheyev, J.S. Williams, C. Jagadish, J. Zou, G. Li, Phys. Rev. B 62, 7510 (2000).

[7] S.O. Kucheyev, J.S. Williams, S.J. Pearton, Mater. Sci. Eng. R 33, 51 (2001). 
[8] F.-R. Ding, W.-H. He, A. Vantomme, Q. Zhao, B. Pipeleers, K. Jacobs, I. Moerman, Mater. Sci. Semicond. Process. 5, 511 (2003).

[9] W. Jiang, W.J. Weber, L.M. Wang, K. Sun, Nucl. Instrum. Meth. Phys. Res. B 218, 427 (2004).

[10] T. Wojtowicz, F. Gloux, P. Ruterana, K. Lorenz, E. Alves, Opt. Mater. 28, 738 (2006).

[11] Y.G. Wang, J. Zou, S.O. Kucheyev, J.S. Williams, C. Jagadish, G. Lie, Electrochem. Solid-State Lett. 6, G34 (2003).

[12] J.B. Gruber, B. Zandi, H.J. Lozykowski, W.M. Jadwisienczak, J. Appl. Phys. 91, 2929 (2002).

[13] B. Pipeleers, S.M. Hogg, A. Vantomme, J. Appl. Phys. 98, 123504 (2005).

[14] K. Lorenz, U. Wahl, E. Alves, T. Wojtowicz, P. Ruterana, S. Ruffenach, O. Briot, Superlattices Microstruct. 36, 737 (2004).

[15] K. Lorenz, E. Alves, U. Wahl, T. Monteiro, S. Dalmasso, R.W. Martin, K.P. O'Donnell, R. Vianden, Mater. Sci. Eng. B 105, 97 (2003).

[16] K. Lorenz, U. Wahl, E. Alves, E. Nogales, S. Dalmasso, R.W. Martin, K.P. O'Donnell, M. Wojdak, A. Braud, T. Monteiro, T. Wojtowicz, P. Ruterana, S. Ruffenach, O. Briot, Opt. Mater. 28, 750 (2006).

[17] C. Stampfl, C.G. Van de Walle, Phys. Rev. B 57, R15052 (1998).

[18] P. Vermaut, P. Ruterana, G. Nouet, Appl. Phys. Lett. 74, 694 (1999).

[19] K. Lorenz, U. Wahl, E. Alves, T. Wojtowicz, P. Ruterana, S. Dalmasso, R.W. Martin, K.P. O‘Donnell, S. Ruffenach, O. Briot, A. Vantomme, Mat. Res. Soc. Symp. Proc. 798, Y5.5.1 (2004).

[20] S.O. Kucheyev, J.S. Williams, J. Zou, C. Jagadish, G. Li, Appl. Phys. Lett. 78, 1373 (2001).

[21] F. Gloux, T. Wójtowicz, P. Ruterana, K. Lorenz, E. Alves, Phys. Status Solidi A 203, 2172 (2006).

[22] S.O. Kucheyev, J.S. Williams, J. Zou, C. Jagadish, M. Pophristic, S. Guo, I.T. Ferguson, M.O. Manasreh, J. Appl. Phys. 92, 3554 (2002).

[23] A.D. Bykhovski, B.L. Gelmont, M.S. Shur, J. Appl. Phys. 78, 3691 (1995).

[24] E. Nogales, R.W. Martin, K.P. O’Donnell, K. Lorenz, E. Alves, S. Ruffenach, O. Briot, Appl. Phys. Lett. 88, 031902 (2006). 